\title{
Sildenafil alters biogenic amines and increases oxidative damage in brain regions of insulin-hypoglycemic rats
}

\author{
DAVID CALDERÓN GUZMÁN ${ }^{1}$ \\ NORMA OSNAYA BRIZUELA ${ }^{1}$ \\ MARIBEL ORTÍZ HERRERA ${ }^{2}$ \\ ARMANDO VALENZUELA PERAZA ${ }^{1}$ \\ GERARDO BARRAGÁN MEJÍA ${ }^{1}$ \\ HUGO JUÁREZ OLGUÍN ${ }^{3,4}$ \\ FRANCISCA TRUJILLO JIMÉNEZ ${ }^{3}$ \\ ${ }^{1}$ Laboratory of Neurosciences \\ Instituto Nacional de Pediatría (INP) \\ Mexico \\ ${ }^{2}$ Laboratory of Experimental Bacteriology \\ INP, Mexico \\ ${ }^{3}$ Laboratory of Pharmacology, INP \\ Mexico \\ ${ }^{4}$ Department of Pharmacology \\ Faculty of Medicine, Universidad \\ Nacional Autónoma de México, Mexico
}

Accepted March 30, 2019

Published May 20, 2019

\begin{abstract}
The aim of the present study was to determine the effect of sildenafil on dopamine, 5-hydroxyindol acetic acid (5-HIAA) and selected biomarkers of oxidative stress in the brain of hypoglycemic rats. The animals were treated intraperitoneally as follows: group 1 (control), saline solution; group 2, insulin (10 $\mathrm{U}$ per rat or $50 \mathrm{U} \mathrm{kg}^{-1}$ ); group 3, insulin + single dose of sildenafil (50 $\left.\mathrm{U} \mathrm{kg}^{-1}+50 \mathrm{mg} \mathrm{kg}^{-1}\right)$; group 4, insulin + three doses of sildenafil every 24 hours $\left(50 \mathrm{U} \mathrm{kg}^{-1}+50 \mathrm{mg} \mathrm{kg}^{-1}\right)$. In groups 2, 3 and 4, insulin was administered every 24 hours for 10 days. Blood glucose was measured after the last treatment. On the last day of the treatment, the animals' brains were extracted to measure the levels of oxidative stress markers $\left[\mathrm{H}_{2} \mathrm{O}_{2}, \mathrm{Ca}^{2+}, \mathrm{Mg}^{2+}\right.$-ATPase, glutathione and lipid peroxidation (TBARS)], dopamine and 5-HIAA in the cortex, striatum and cerebellum/medulla oblongata by validated methods. The results suggest that administration of insulin in combination with sildenafil induces hypoglycemia and hypotension, enhances oxidative damage and provokes changes in the brain metabolism of biogenic amines. Administration of insulin and sildenafil promotes biometabolic responses in glucose control, namely, it induces hypoglycemia and hypotension. It also enhances oxidative damage and provokes changes in the brain metabolism of biogenic amines.
\end{abstract}

Keywords: sildenafil, rat, hypoglycemia, biogenic amines, glutathione, $\mathrm{H}_{2} \mathrm{O}_{2}$, lipid peroxidation

Obesity increases the risk of atherosclerosis, renal diseases, vesicular lithiasis and diabetes. In the last few decades, type 2 diabetes mellitus has reached epidemic proportions due to bad lifestyles (1). In recent times, multiple drugs for effective management of obesity have emerged but most of them confer only modest benefits for patients. The majority of patients require insulin to control diabetes; however, insulin may provoke hypoglycemic conditions in patients with this pathology (2). Erectile dysfunction (ED) associated with type 2 diabetes is a severe problem, which requires effective treatment (3). Most adult patients, irrespective of age, will require sildenafil as an option for this dysfunction and it

\footnotetext{
* Corresponding author; e-mail: juarezol@yahoo.com
} 
has recently become the preferred treatment of choice (4). Effects of sildenafil on the pulmonary vasculature and on erectile dysfunction are increasingly well understood (5); nevertheless, its mechanism in hypoglycemic patients is still unknown.

On the other hand, obesity and overweight have been associated with an increase in free radicals (FR) (6), which can put the cells of the central nervous system (CNS) in danger of oxidative damage caused by excessive production of oxygen reactive species involved in many pathological processes (7). Oxidative stress dysregulates energy and glucose homeostasis in different brain regions and disrupts brain circuits such as the dopaminergic system (8).

Plasma membrane phospholipids in neurons are in close contact with structural proteins embedded in the lipid bilayer, from which $\mathrm{Na}^{+}, \mathrm{K}^{+}$-ATPase is responsible for keeping the ionic interchange through this bilayer by stimulating $\mathrm{Na}^{+}$and $\mathrm{K}^{+}$flows. Inhibition of $\mathrm{Na}^{+}, \mathrm{K}^{+}$-ATPase activity alters the ionic interchange within the CNS (9).

In view of the above, the purpose of the present study was to evaluate the effect of sildenafil on the levels of dopamine and 5-HIAA monoamines, and selected oxidative stress markers in brain regions of young rats with insulin induced hypoglycemia.

\section{EXPERIMENTAL}

The study included twenty-eight male Wistar rats of 7 weeks of age and $200 \mathrm{~g}$ mean body mass. During the study, all animals were kept in closed boxes under a light:dark period of 12:12 h. Animals were divided into four groups of 7 rats each and treated intraperitoneally as follows: group 1 (control), $0.9 \% \mathrm{NaCl}$; group 2, insulin (10 U per rat or $\left.50 \mathrm{U} \mathrm{kg}^{-1}\right)$; group 3, insulin $\left(50 \mathrm{U} \mathrm{kg}^{-1}\right)+$ single dose of sildenafil $\left(50 \mathrm{mg} \mathrm{kg}^{-1}\right)$; group 4, insulin $\left(50 \mathrm{U} \mathrm{kg}^{-1}\right)+$ three doses of sildenafil ( $50 \mathrm{mg} \mathrm{kg}^{-1}$ ) every 24 hours. Treatment groups received insulin every 24 hours for 10 days. Groups $2-4$ were treated every day with insulin for 10 days. Group 3 received sildenafil once, on day 10 . Group 4 received sildenafil three times, namely, on days 8,9 and 10, each 24 hours. Sixty minutes after receiving the last treatment, animals' blood was taken from the tail and used to measure their glucose levels. Animals were sacrificed by decapitation without anesthesia. Brains were extracted and immediately placed in a 0.9 $\%$ cold $\mathrm{NaCl}$ solution. They were later divided into the cortex, cerebellum/medulla oblongata and striatum. Tissues from these brain regions were then homogenized in a $0.05 \mathrm{~mol} \mathrm{~L}^{-1}$ phosphate buffer solution (PBS) and used to quantify 5-hydroxyindol acetic acid (5-HIAA), dopamine, $\mathrm{Ca}^{2+}, \mathrm{Mg}^{2+}$-ATPase, $\mathrm{H}_{2} \mathrm{O}_{2}$, lipid peroxidation and glutathione (GSH) using previously validated methods (10). All samples were frozen at $-20^{\circ} \mathrm{C}$ until analysis.

Animal experiments were carried out in strict compliance with the Guidelines for Ethical Control and Supervision in the Care and Use of Animals and all experimental procedures were conducted following national and international rules. Also, the study was approved by the Laboratory Animals Use and Care Committee of the National Institute of Pediatrics, Ciudad de México, México.

\section{Statistical analysis}

Two-way ANOVA and Tukey's test were used in the analysis of the results while the statistical Dunnett's test was employed for Kramer HSD (honestly significant difference) 
for comparison of all pairs. For all tests, values of $p<0.05$ were considered statistically significant. Statistical Software JMP version 8.0.0 for academic purposes was used.

\section{RESULTS AND DISCUSSION}

Administration of insulin alone or in combination with sildenafil in single or repeated doses decreased the levels of glucose in a significant way $(p<0.0001)$ compared to the control group. Comparison among the experimental groups showed that the group treated with insulin + sildenafil in repeated doses had the biggest decrease in glucose levels, which in the group treated with insulin alone showed to be significantly different (Table I).

Table I. Levels of glucose in the blood of animals treated intraperitoneally either with insulin, sildenafil or both

\begin{tabular}{cc}
\hline Group/treatment & Glucose (mg per 100 mL) \\
\hline Control (saline) & $110.50 \pm 7.56$ \\
Insulin $\left(50 \mathrm{U} \mathrm{kg}^{-1}\right)$ & $57.83 \pm 4.96^{*}$ \\
Insulin $\left(50 \mathrm{U} \mathrm{kg}^{-1}\right)+$ sildenafil $\left(50 \mathrm{mg} \mathrm{kg}^{-1}\right)-$ single dose & $48.57 \pm 7.50^{*}$ \\
Insulin $\left(50 \mathrm{U} \mathrm{kg}^{-1}\right)+$ sildenafil $\left(50 \mathrm{mg} \mathrm{kg}^{-1}\right)-3$ doses & $46.57 \pm 4.04^{*, * *}$ \\
\hline
\end{tabular}

Average $\pm \mathrm{SD}, n=7$.

Statistically significant difference: ${ }^{*} p<0.0001 v$ s. control, ${ }^{* *} p=0.017 v$ s. insulin.

In the cortex, administration of insulin alone or combined with sildenafil produced a significant decrease in the levels of lipid peroxidation (TBARS - thiobarbituric acid reactive substances) compared to the control group (Table II).

A similar pattern was observed in the striatum; however, only in group 4 treated with repeated doses of sildenafil, TBARS level was significantly different from the control group although it was not different at all from the groups treated with insulin or a single dose of insulin + sildenafil (Table III).

In the cerebellum/medulla oblongata, a significant decrease was observed in the groups treated with a combination of insulin and sildenafil, both in single and repeated doses, compared to the control (Table IV). Similarly to what was observed in the cortex, the group that received repeated doses of insulin + sildenafil showed a larger decrease in the level of TBARS, which was significantly different from the group treated with a single dose of insulin + sildenafil (Table II).

In the cortex, groups 2 and 3 showed a significant decrease in the levels of $\mathrm{H}_{2} \mathrm{O}_{2}$; in the striatum, however, a discrete increase which was not significant was observed in the experimental groups compared to the control group. With regard to cerebellum/medulla oblongata, all groups showed a significant decrease in the levels of $\mathrm{H}_{2} \mathrm{O}_{2}$ compared to the control. Again, group 4 treated with repeated doses of sildenafil manifested a greater decrease in $\mathrm{H}_{2} \mathrm{O}_{2}$ levels, which was significant compared to the group treated with insulin alone. 


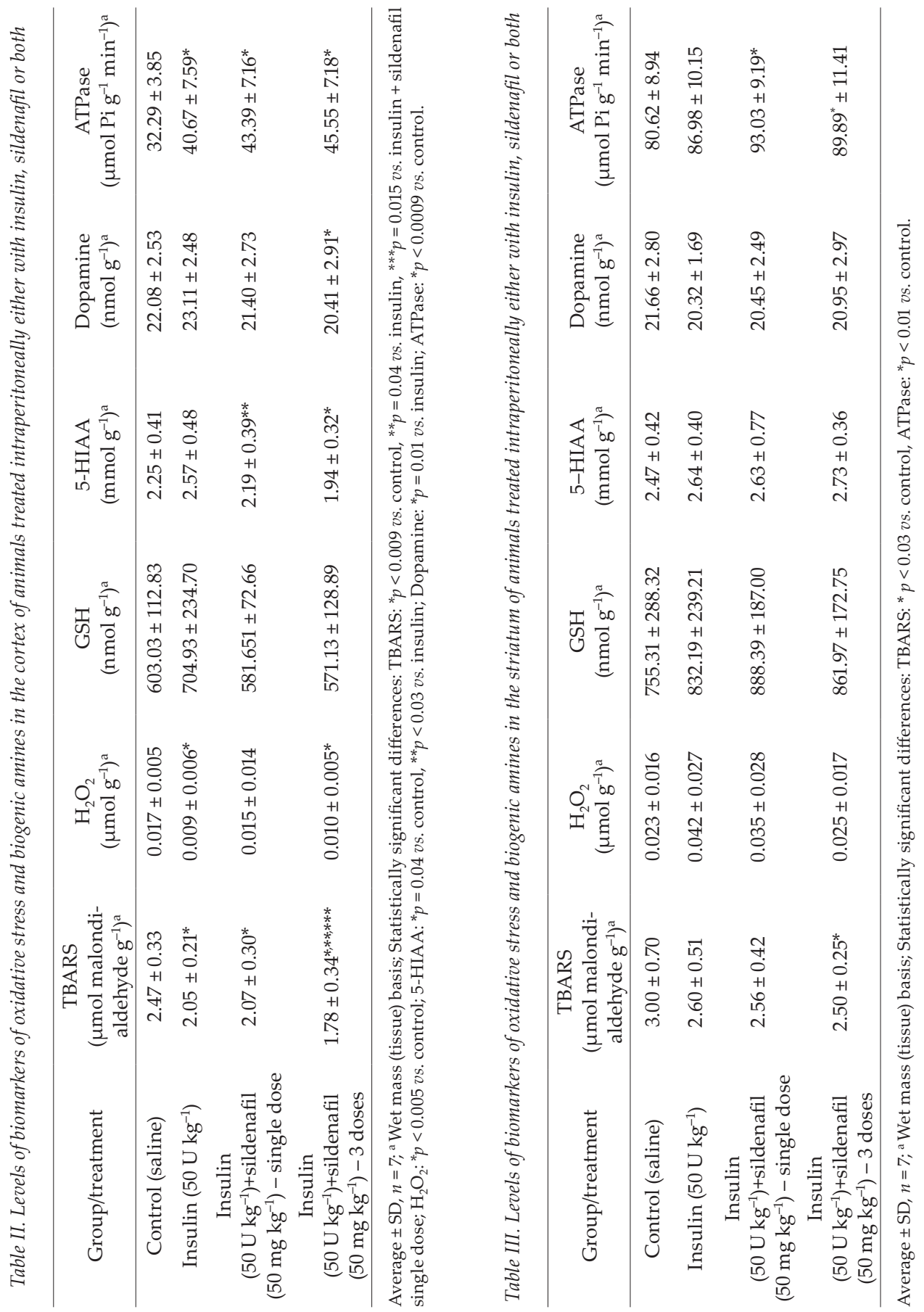


In the cortex and striatum, statistical analysis of the effects of insulin alone or combined with single or repeated doses of sildenafil on the levels of GSH did not reveal significant changes compared to the control group. In the cerebellum/ medulla oblongata, a significant increase was observed in the levels of this biogenic amine in the groups treated with insulin alone and group 4 with repeated doses of sildenafil, compared to the control group.

Statistical analysis of the data revealed a significant increase in the levels of 5-HIAA in the cortex of group 4 treated with insulin + repeated doses of sildenafil compared to the control. Groups treated with the combination of insulin + sildenafil in both treatment schemes showed a decrease in the levels of 5-HIAA compared to the group that received insulin alone. In the striatum and cerebellum/ medulla oblongata, the analysis did not reveal any significant changes in the concentration of 5-HIAA. Administration of insulin + sildenafil in repeated doses produced a decrease in the concentration of dopamine (DA) in the cortex, which was statistically significant only compared to the group that received insulin alone. In other regions of the brain, no changes in the concentration of DA were observed.

In the case of ATPase activity, the administration of insulin alone or combined with sildenafil increased significantly the activity of amine in the cortex and striatum. However, in the cerebellum/ medulla oblongata, this increase was statistically significant only for group 4 treated with insulin + repeated doses of sildenafil compared to the control and the group that received a single dose of insulin + sildenafil.

A major issue in glycemic control in neurocritical care is the lack of continuous glucose monitoring in patients under insulin therapy. Thus, these patients are 
associated with higher rates of hypoglycemia without a chance to improve their survival rate. This may probably be the reason why Bilotta and Rossa (11) recommended adequate nutrition before and during insulin infusion.

In this work, dopamine and 5-HIAA led to a significant decrease in the cortex regions. These findings coincide with that of Ammon-Treiber et al. (12) who suggest that decreased levels of DA and 5-HIAA can aggravate neurotoxic effects on the hypoxia/hypoglycemia model, and that their metabolites may increase oxidative damage to the brain.

Hypoglycemic animals exerted a decrease in the levels of glutathione, suggesting that insulin and sildenafil provide no protection from oxidative stress to the brain. Hence, such doses of insulin and sildenafil may make the CNS vulnerable to damage by oxidative stress caused by depletion of the glutathione antioxidant. NO production in selected neuron populations may have induced oxidative stress (13), which altered GSH metabolism in the brain.

Suppression of reactive oxygen species-dependent pathways in the endothelium restores insulin delivery to peripheral organs by preserving nitric oxide (NO) availability. The lipoperoxidation and $\mathrm{H}_{2} \mathrm{O}_{2}$ levels decreased as a consequence of sildenafil and insulin treatment. This result is in accord with what was previously reported in our laboratories where hypoglycemia was associated with increased oxidative damage (14).

Animals treated with insulin and sildenafil experienced an increase in total ATPase in the cortex and striatum. This finding agrees with the report of Filippov (15), who suggested an increase in the number of active molecules of ATPase under the influence of membrane structural changes in the hypoglycemic state. There was a decrease in blood glucose in the animals that received sildenafil, thus confirming the hypoglycemia model and a potential increase of oxidative damage with the use of this drug. This concurs with Mandosi et al. (16) who found out that sildenafil exerted a beneficial effect on glycometabolic control in type 2 diabetes by increasing insulin sensitivity (17). Moreover, the combination of sildenafil and insulin may offer an improved cerebrovascular response (18), due to its relaxation action on corpora cavernosa.

\section{CONCLUSIONS}

Administration of insulin and sildenafil promoted biometabolic responses in glucose control. Hypoglycemic animals showed a decrease in the levels of glutathione suggesting that insulin and sildenafil provided no protection from oxidative stress to the brain. Hence, such doses of insulin and sildenafil may make the CNS vulnerable to damage caused by oxidative stress due to depletion of the glutathione antioxidant.

\section{REFERENCES}

1. A. V. Raveendran, E. C. Chacko and J. M. Pappachan, Non-pharmacological treatment options in the management of diabetes mellitus, Eur. Endocrinol. 14 (2018) 31-39; https://doi.org/ 10.17925/ EE.2018.14.2.31

2. A. Hussain, O. B. Latiwesh, F. Ali, M. Y. G. Younis and J. A. Alammari, Effects of body mass index, glycemic control, and hypoglycemic drugs on serum uric acid levels in type 2 diabetic patients, Cureus 10 (2018) e3158; https://doi.org/ 10.7759/cureus.3158 
3. G. T. Chen, B. B. Yang, J. H. Chen, Z. Zhang, L. L. Zhu, H. S. Jiang, W. Yu, Y. Chen and Y. T. Dai, Pancreatic kininogenase improves erectile function in streptozotocin-induced type 2 diabetic rats with erectile dysfunction, Asian J. Androl. 20 (2018) 448-453; https://doi.org/ 10.4103/aja.aja_23_18

4. L. Chen, S. E. Staubli, M. P. Schneider, A. G. Kessels, S. Ivic, L. M. Bachmann and T. M. Kessler, Phosphodiesterase 5 inhibitors for the treatment of erectile dysfunction: a trade-off network meta-analysis, Eur. Urol. 68 (2015) 674-680; https://doi.org/ 10.1016/j.eururo.2015.03.031

5. R. S. Calabrò, G. Polimeni and P. Bramanti, Current and future therapies of erectile dysfunction in neurological disorders, Rec. Pat. CNS Drug Discov. 6 (2011) 48-64.

6. S. J. Flora, Role of free radicals and antioxidants in health and disease, Cell. Mol. Biol. 53 (2007) 1-2.

7. J. T. Coyle and P. Puttfarcken, Oxidative stress, glutamate, and neurodegenerative disorders, Science 262 (1993) 689-695.

8. M. C. Vogt and J. C. Brüning, CNS insulin signaling in the control of energy homeostasis and glucose metabolism - from embryo to old age, Trends Endocrinol. Metab. 24 (2013) 76-84; https://doi. org/10.1016/j.tem.2012.11.004

9. G. D. Calderon, N. Osnaya Brizuela, M. Ortiz Herrera, O. H. Juarez, A. Valenzuela Peraza and G. Barragan Mejía, Effect of an antiviral and vitamins A, C, D on dopamine and some oxidative stress markers in rat brain exposed to ozone, Arch. Biol. Sci. Belgrade 65 (2013) 1371-1379; https:// doi.org/10.1186/s12868-018-0474-4

10. D. Calderón Guzmán, N. Osnaya Brizuela, M. Ortíz Herrera, E. Hernandez Garcia, G. Barragan Mejía, H. Juarez Olguín, A. Valenzuela Peraza, J. Attilus and N. Labra Ruiz, Effect of cerebrolysin on dopaminergic neurodegeneration of rat with oxidative stress induced by 3-nitropropionic acid, Acta Pharm. 66 (2016) 443-448; https://doi.org/10.1515/acph-2016-0027

11. F. Bilotta and G. Rosa, Optimal glycemic control in neurocritical care patients, Crit. Care 16 (2012) 163-165; https://doi.org/10.1186/cc11521

12. S. Ammon-Treiber, D. Stolze, H. Schroder, H. Loh and V. Hollt, Effects of opioid antagonists and morphine in a hippocampal hypoxia/hypoglycemia model, Neuropharmacology 49 (2005) 11601169; https://doi.org/10.1016/j.neuropharm.2005.06.016

13. J. H. Jhamandas, K. H. Harris, T. Petrov and K. H. Jhamandas, Activation of nitric oxidesynthesizing neurons during precipitated morphine withdrawal, Neuroreport 7 (1996) 2843-2846.

14. G. D. Calderón, G. E. Hernández, M. G. Barragán, O. H. Juárez, G. J. Saldivar and R. N. Labra, Effect of morphine and lacosamide on levels of dopamine and 5-HIAA in brain regions of rats with induced hypoglycemia, Pak. J. Biol. Sci. 17 (2014) 292-296.

15. S. P. Filippov, ATPase activity of rat brain microsomal and synaptosomal fractions in insulin hypoglycemia and its treatment with glucose, Probl. Endokrinol. (Moskow) 37 (1991) 52-54; https:// doi.org/10.1134/S0022093006020062

16. E. Mandosi, E. Giannetta, T. Filardi, M. Lococo, C. Bertolini, M. FAllarino, D. Gianfrilli, M. A. Venneri, L. Lenti, S. Morano and A. Lenzi, Endothelial dysfuntion markers as a therapeutic target for sildenafil treatment and effects on metabolic control in type 2 diabetes, Expert Opin. Ther. Target. 19 (2015) 1617-1622; https://doi.org/10.1517/14728222.2015.1066337

17. C. E. Ramirez, H. Nian, Y. Chang, L. J. Gamboa, J. M. Luther, N. J. Brown and C. A. Shibao, Treatment with sildenafil improves insulin sensitivity in prediabetes: a randomized, controlled trial, JCEM 100 (2015) 4533-4540; https://doi.org/10.1210/jc.2015-3415

18. F. G. Al-Amran, A. A. Zwain, N. R. Hadi and A. M. Al-Mudhaffer, Autonomic cerebral vascular response to sildenafil in diabetic patient, Diabetol. Metab. Syndr. 4 (2012) 2-8; https://doi. org/10.1186/1758-5996-4-2 